\title{
Bourse de recherche
}

Beaufour Ipsen Pharma, impliqué depuis de nombreuses années dans le développement des connaissances en colo-proctologie, met à la disposition des médecins, chirurgiens, étudiants en médecine spécialisés en gastro-entérologie, proctologie, chirurgie digestive et viscérale, une Bourse de Recherche en Colo-proctologie d'un montant de 12500 euros, en partenariat avec la Société Nationale Française de Colo-Proctologie.

Cette bourse, décernée à un candidat unique, doit permettre ou aider à la réalisation d'un projet original clinique, pharmaco-clinique ou thérapeutique en Colo-proctologie.

Comme chaque année, la remise de cette bourse aura lieu à l'occasion des Journées Francophones de Pathologie Digestive à Paris qui se dérouleront du 20 au 22 mars 2006.

Le règlement de cette bourse est disponible auprès du :

\section{Département de Colo-Proctologie de Beaufour Ipsen Pharma}

24, Rue Erlanger - 75781 Paris Cedex 16

Tél. 01-44-96-13-13

La date limite de dépôt des dossiers est fixée au $\mathbf{1 5}$ février $\mathbf{2 0 0 6 .}$ 